\title{
Mice, like humans, move in fits and starts
}

Until fairly recently, human activity was not thought to follow a particular pattern. Human actions are subject to personal decisions, and these are presumed to be too complex to predict reliably. Yet studies from the past few years have shown that contrary to this assumption, human activity — ranging from general movement to the execution of specific tasks such as sending e-mail and browsing the web-fits a consistent organizational structure of bursts of activity followed by periods of inactivity. The distribution and duration of resting and active periods are highly conserved across individuals.

A new study by Yoshiharu Yamamoto (University of Tokyo, Japan) and colleagues shows that these behavioral patterns are not limited to humans but apply to mice, as well (PLoS ONE 3, e2050; 2008). This finding suggests the possibility of a single principle governing the behavior of mammalian species and may have meaningful implications for behavioral modeling.

Yamamoto and colleagues had previously collected data on the activity of human adults. In this study they recorded the movements of healthy adolescents who

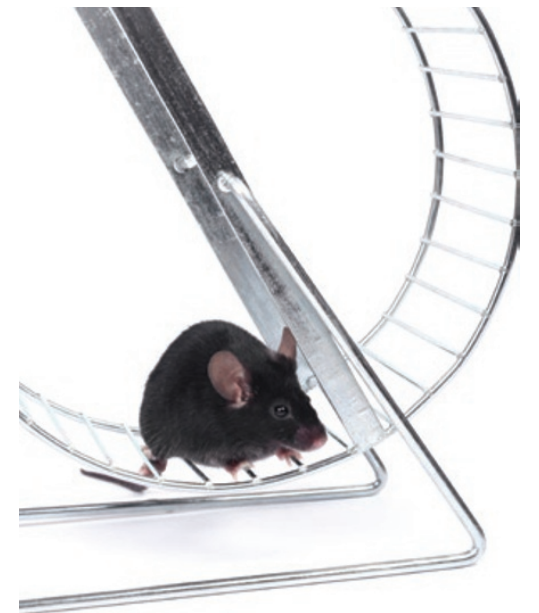

they found that the organization of activity was identical between the species.

The researchers then explored a potential application of these findings. Reduction in locomotor activity and disruption of the circadian cycle are among the key signs of clinical depression. Yamamoto and colleagues had previously shown that the activity pattern of depressed adults deviated from that of healthy adults. In this study the researchers compared historical data from depressed humans with new activity data from knockout mice lacking . the normal rest-activity cycle. The behavioral patterns were identical.

It is too soon to conclude that the mutant mice can model depression,

wore watch-like monitoring devices for several days. The researchers also monitored the activity of mice using pressure-sensing sheets that were placed under mouse cages.

The group showed first that activity patterns of adolescents were the same as those of adults, despite their markedly different lifestyles. When the researchers compared data from healthy humans with rescaled data from wild-type mice, Yamamoto tells Lab Animal. Rather, the importance of this study lies in the "demonstration of 'universality' in how animals move." The shared behavioral organization may offer an opportunity to "study objectively the relationship between a symptom of neurobehavioral disorders, major depression in this case, and a symptom associated with elimination of a specific gene."

Karen Marron

\section{SUGARS CLICK WITH ZEBRAEISH}

New advances in molecular tagging may one day offer deeper insights into the development of diseases such as cancer and heart disease. Now in its infancy, the technique has been applied to tracking the development of the zebrafish embryo in vivo using noninvasive imaging of sugar molecules.

Sugars at the cell surface contain much information about a cell's physiological state. For example, changes in their structures may act as markers of changes in gene expression that occur during cell development and disease progression. Therefore, these sugars are prime targets for diagnostic in vivo imaging, but have so far been poor candidates because they are incompatible with genetically encoded reporter molecules.

The new technique is the latest advance in 'click chemistry', or the linking of molecules that are otherwise not readily joined together. A popular version of these reactions uses a copper catalyst to link azides, small chemical groups that are stable in vivo, with activated alkynes. The azide groups are attached to sugars, which are then incorporated into the cells of interest. In the presence of copper, the azides react with fluorescently labeled alkynes, allowing researchers to track the sugars. But copper is toxic to living organisms, limiting the use of this technique to ex vivo applications.
To enable in vivo imaging of the sugars, Carolyn R. Bertozzi and colleagues at the University of California Berkeley modified the reaction by eliminating the need for the copper catalyst. They achieved this by bending the alkynes into rings, allowing them to react quickly with the azides without requiring a catalyst. Bertozzi and colleagues previously reported on the use of this technique to stain rodent tissue samples.

Now, they have taken the next step and used the technique to track cell-surface sugars during embryonic development in vivo in zebrafish (Science 320, 664-667; 2008). The zebrafish was a good candidate for this study because its development is well-documented, it is frequently used in biomedical research, and it is amenable to optical imaging. Bertozzi's group was able to simultaneously track the distribution of different sugar groups throughout embryonic development and detect patterns that could not have been observed using conventional molecular imaging methods. They recorded bursts of sugar synthesis in the jaw, pectoral fins and olfactory organs 60 hours after fertilization.

So far the technique has been used in rodents and in fish, and the researchers believe it will function in other organisms as well. Future studies will aim to apply the technique to tracking cells in tumors and diseased blood vessels.
Monica Harrington 